\title{
A FADING CHARACTER OF WHISTLER TRACES DISPLAYED ON SONAGRAM
}

\author{
Sazanami Yano \\ Department of Electrical Engineering \\ Kochi National College of Technology, Nangoku 783 \\ Toshio Ogawa \\ Science Laboratory International \\ 2C Sunset Building, 1510-1 Kamobe, Kochi 780 \\ Hiroshi Takano \\ Department of Mechanical Engineering \\ Kochi National College of Technology, Nangoku 783 \\ Mikio Fujito and Yasuo Seki \\ Department of Electrical Engineering \\ Kochi National College of Technology, Nangoku 783
}

\begin{abstract}
A fading character of whistler traces has been studied (Yano et al.,1989). During the course of this study it is noticed that quick fading patterns of whistler waves obtained by a sound-spectrograph, sonagraph, show an inflation such as semi-circular shift to the right. In order to explain this phenomenon the fading of two artificial whistlers generated by using a digital computer and a D/A converter was analyzed by the sonagraph. It is concluded as a result of the experiment that the rightward inflation of the trace is caused by the resonance echo of a high- $Q$ bandpass filter mounted on the sonagraph.
\end{abstract}

\section{Introduction}

Shaw and Gurnett (1971) reported on 'harmonic bands' observed on a whistler in the Injun 5 satellite data. In their data the average frequency difference between the adjacent bands showed 15.7 $\mathrm{Hz}$. They concluded that the harmonic bands were produced by interference between multiple lightning strokes. Paymar (1972) reported on the 'banded' structure of whistler traces observed on OGO-4 satellite with the dispersion of around $90 \mathrm{sec}^{1 / 2}$. These banded whistlers were observed at around $40^{\circ}$ geomagnetic latitude with the low latitude cutoff at $\sim 30^{\circ}$, and were explained as that they were caused by a mechanism of spectral reflection or transmission of the irregularities with an enhanced ionization in an ambient plasma. Ohta et al.(1988) reported on 'banded' structure of contour lines of the intensity in the Fourier spectra of whistler traces obtained by using the FFT digital processor. They explained that this structure was produced by the wave interference in a horizontally stratified irregularity in the $\mathrm{F}$ region ionosphere of down-coming whistler waves. Ohta et al.(1989) also called the same structure of the trace the 'patched' structure. Yano et al.(1989) observed similar kind of phenomena and pointed out that the amplitude modulation of a whistler wave can be made by the interference of two or more whistler waves which are propagating after making different hops in the Earth-ionosphere waveguide. They called this phenomenon the 'fading'. There are quick and slow fading patterns displayed on the sonagram. In the quick fading the trace seems to shift rightward, and it was suggested that this rightward inflation of the wave trace may be caused by the resonance echo of a high $Q$ bandpass filter mounted on the sonagraph without showing the background. In this paper we discuss details of the cause of this phenomenon. 


\section{Experiment}

Figure 1 shows two examples of sonagrams of whistler traces exhibiting fading phenomena observed at Kochi. Figures 1 (a) and (b) show a quick fading trace between two slow fading traces. The traces " $A$ ", " $B$ ", and " $C$ " have the same dispersion value of $26 \sec ^{1 / 2}$ which is typical at Kochi. The three whistlers were observed within a half second. The traces $A$ and $C$ show relatively slow fading, while the trace $B$ shows quick fading. Figures 1 (c) and (d) show hybrid patterns with quick and slow fading. In these figures, the traces with the quick fading show the rightward inflation.

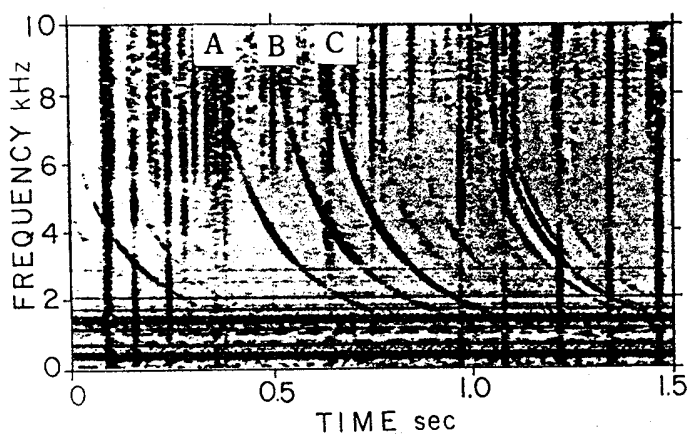

(a)

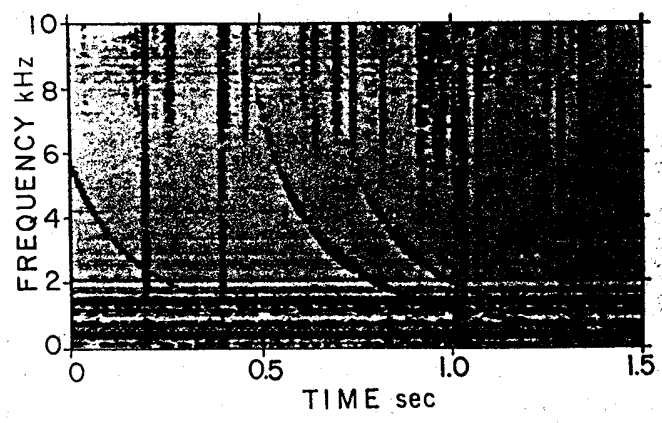

(c)

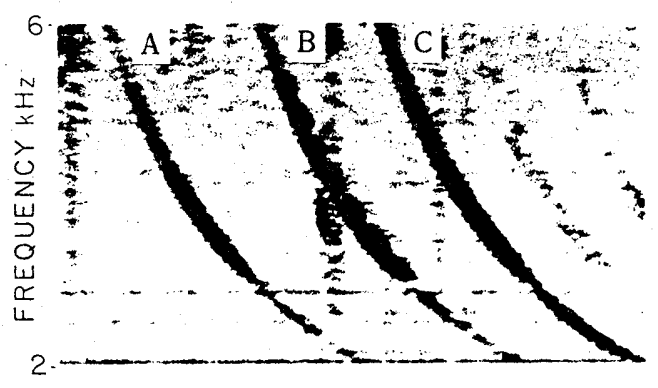

TIME

(b)

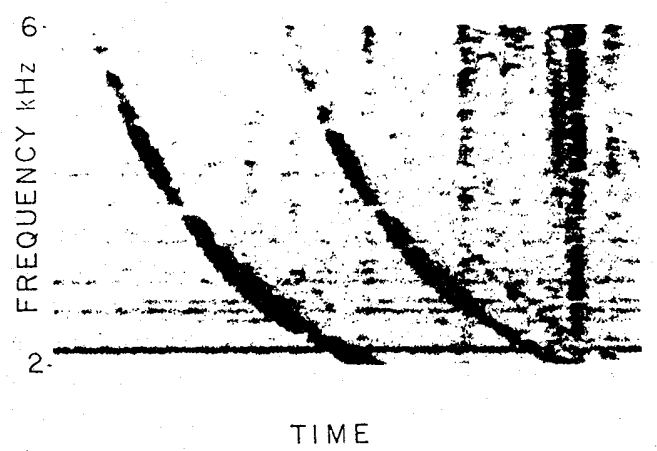

(d)

Fig.1. Sonagrams of whistler traces with the rightward inflation in the quick fading patterns. (a) Quick fading trace with rightward inflation observed between slow fading traces. (b) Magnification of the inflating pattern of (a). (c) Hybrid patterns with quick and slow fading with the rightward inflation. (d) Magnification of the inflating pattern of (c).

To show a possible mechanism of the rightward inflation of the traces, the waves generated by the next equations in a digital computer are used.

$$
e_{n}=m_{n} A_{1} \cos \left\{\omega_{n}\left(t+\Delta t_{n}\right)\right\}, \quad \omega_{n}=2 \pi \quad\left\{D /\left(t+\Delta t_{n}\right)\right\}^{2}
$$

where $A_{1}$ is the amplitude of the first wave, $m_{n}$ is the amplitude ratio of $n$-th wave to the first, $D$ is the dispersion constant, and $\Delta t_{n}$ is the time delay of the $n$-th wave from the first. A whistler wave with fading is given by the summation of these $n$ waves (Yano et al.,1989). Then the calculation formula of the artificial whistler is obtained as following. 


$$
e=\sum_{n=1}^{N} e_{n}
$$

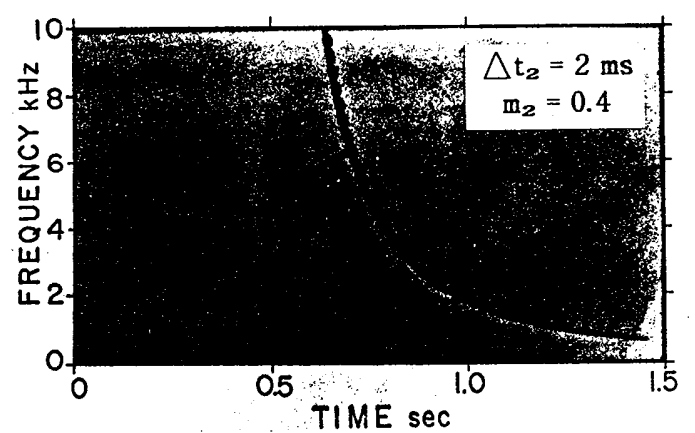

(a)

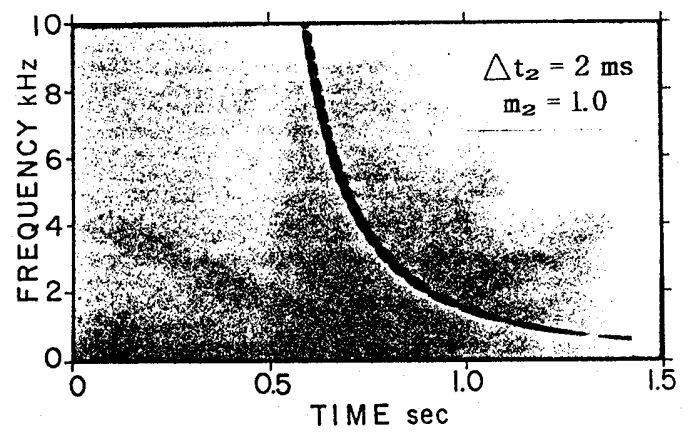

(c)

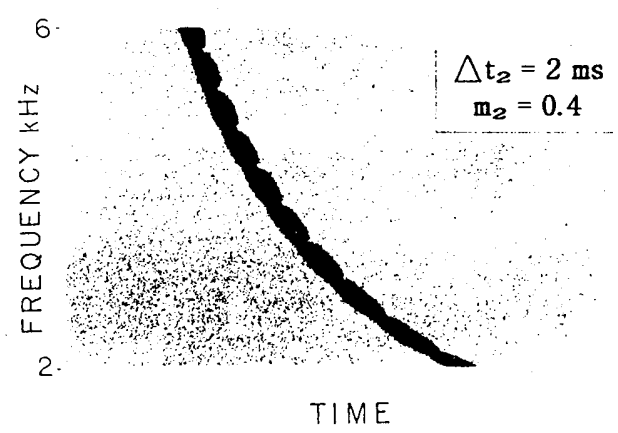

(b)

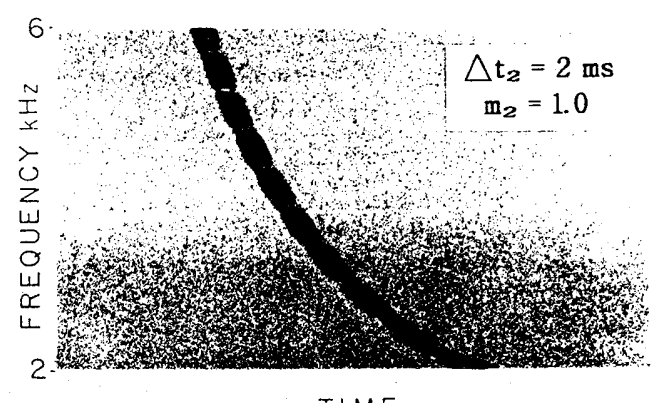

TIME

(d)

Fig.2. Sonagrams of artificial whistlers. (a) A trace showing the rightward inflation of the fading pattern of two waves calculated with $\Delta \mathrm{t}_{2}=2 \mathrm{~ms}$ and $\mathrm{m}_{2}=0.4$. (b) Magnification of the inflating pattern of the trace in (a). (c) A trace showing the rightward inflation of the fading pattern of two waves calculated with $\Delta t_{2}=2 \mathrm{~ms}$ and $\mathrm{m}_{2}=1.0$. (d) Magnification of the pattern of (c).

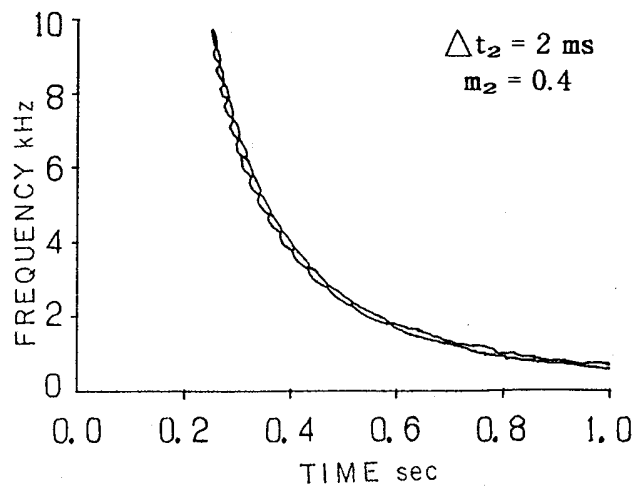

(a)

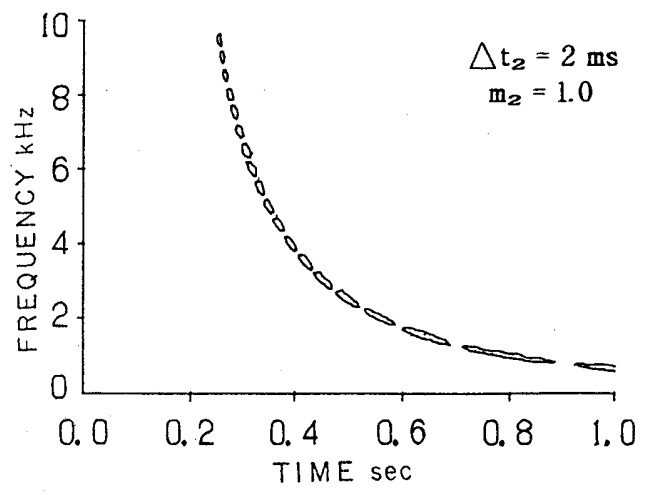

(b)

Fig.3. Frequency-to-time curves obtained by analyzing the same data as in Fig. 2 by a FFT processor on a digital computer. (a) and (b) show the contour lines of equal intensity on the fading pattern of two waves with $\Delta t_{2}=2 \mathrm{~ms}$, and $\mathrm{m}_{\mathbf{z}}=0.4$ and 1.0 , respectively. 
The waves calculated by using Eq.(2) are converted to an analogue signal through a D/A converter and recorded on the magnetic tape. Then the artificial whistler sound obtained by reproducing from the tape is analyzed by the sonagraph. To keep the same situation as a treatment of the measured whistler, the same magnetic tape recorder is used as a recording apparatus for both of the measured and the artificial whistlers.

Figure 2 gives two examples of sonagrams showing the fading pattern made by two artificial whistlers. Figures 2 (a) and (b) show a trace with the amplitude ratio $\mathrm{m}_{2}=0.4$ and the delay time $\triangle t_{2}=2 \mathrm{~ms}$. We can see the rightward inflation phenomena on each loop of the fading pattern on the trace. Figures 2 (c) and (d) show a trace of $\mathrm{m}_{2}=1.0$ and $\Delta \mathrm{t}_{2}=2 \mathrm{~ms}$. In this case, the pattern structure looks like a train of the patches or a banded structure rather than like a 'rightward inflation'. The rightward inflation of the trace becomes clearer as the fading frequency becomes higher. As the amplitude of two whistler waves becomes closer to each other, the trace shows the structure like a train of patches or a banded type.

Figure 3 shows the $f-t$ curves of the artificial whistlers which are displayed on the digital computer by calculating Eq.3, and by processing the signals with FFT. Figures 3 (a) and (b) show the contour lines of the intensity of the fading pattern with $D=26 \mathrm{sec}^{1 / 2}$ of the waves with $\triangle t_{2}$ $=2 \mathrm{~ms}$, and $\mathrm{m}_{2}=0.4$ and 1.0 , respectively. As are seen in these figures, the fading pattern do not show the rightward inflation of the traces.

\section{Discussion}

In Figs. 2 (a) and (b), the trace of the artificial whistler analyzed by the sonagraph shows the fading patterns with the rightward inflation, whereas the $f-t$ curves made by the digital computer in Fig. 3 do not show the rightward inflation. Therefore the rightward inflation phenomenon may be made in the sonagraph. In the sonagraph, the time delay which depends on the wave amplitude is caused by a bandpass filter mounted in the machine. Thus it can be said that the rightward inflation of the traces obtained by using the sonagraph may be caused by the resonance echo in the high- $Q$ bandpass filter.

The semi-circular rightward inflation of the trace seems to appear when the amplitude ratio $\mathrm{m}_{2}$ of two waves is less than 0.5 and the delay time $\Delta t_{2}$ is around 2 ms. In a comparison between Fig.l and Fig.2, we see the larger rightward inflation in the measured whistler trace than in the artificial whistler. A reason to cause the larger inflation in the measured ones than in the artificially computed may be caused by a perturbation of the propagation constant in a whistler duct in the magnetosphere.

The traces in Figs. 2 (c) and (d), on the other hand, show a banded structure or a train of patches rather than the rightward inflation, and are caused by the fading of two or more whistier waves with nearly the same amplitude such as $m_{n}=1.0$.

Figure 4 shows the schematic diagram of the electronic circuit to measure the voltage response of a mechanical bandpass filter. The FM signal with a decreasing frequency at the input terminal of the bandpass filter (BPF) is obtained by the switch (S. W.) being off. The waveformes at the output of the BPF and on the cathode ray tube are shown in the upper portion of the diagram, respectively.

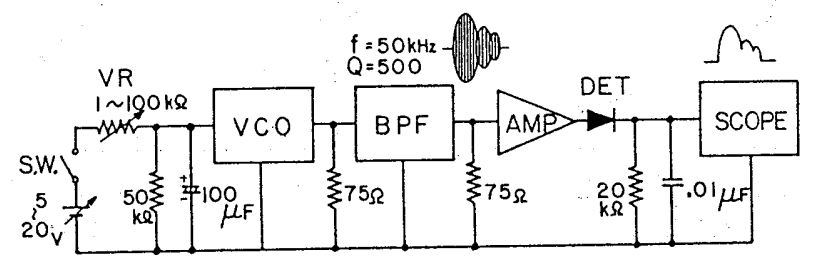

Fig. 4. Schematic diagram to measure the voltage response of a mechanical bandpass filter when the input voltage with a decreasing frequency is applied. 
Figure 5 shows the envelope of the output voltage of the high-Q mechanical filter with $Q=250$ at $\mathrm{f}=50 \mathrm{kHz}$ when the FM signal with the decreasing frequency is applied to the input of the circuit shown in Fig. 4. Figures 5 (a) and (b) are obtained with the input voltages of $0.2 \mathrm{Vpp}$ and $20 \mathrm{Vpp}$, respectively. An extra peak signal can be seen in Fig. 5 (a) and 4 extra peak signals in Fig.5 (b) after the respective main peaks. These extra signals originate in the resonance of a resonator at the frequencies of attenuating range and they are so called the resonance echo. It can be seen in Fig. 5 that the resonance echo depends on the signal amplitude. As the amplitude of the input voltage increases, the echo peaks glow in both the relative amplitude and the number. Furthermore it became clear during the course of this measurement that as the time differential of the local frequency of a signal becomes larger, the relative amplitude and number of the echo peaks become larger.

Figure 6 is a sketch to show a mechanism how the BPF makes the pattern with the rightward inflation. As the amplitude of the signal becomes larger, the trace on the sonagram becomes to have a wider pattern with higher, echos.

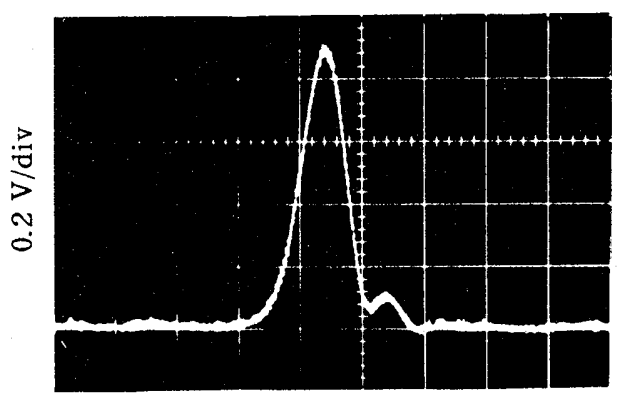

$10 \mathrm{~ms} / \mathrm{div}$

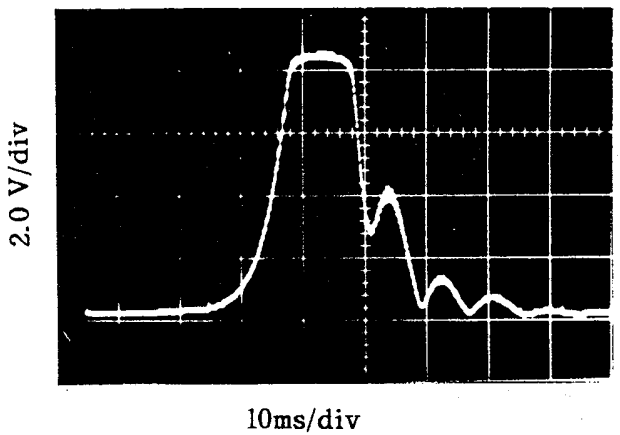

(b)

Fig.5. The wave envelope showing the resonance echo generated in the high- $Q$ mechanical bandpass filter when the input voltage with decreasing frequency is applied. (a) and (b) show the response for $0.2 \mathrm{Vpp}$ and $20 \mathrm{Vpp}$ of the input voltage, respectively.

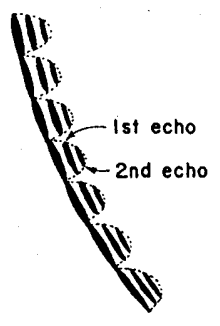

Fig.6. A sketch to show how the rightward inflation is made on a trace in the sonagram.

These figures explain that if one uses a spectrogragh with a high- $Q$ mechanical filter, then will meet with the resonance echo phenomena.

\section{Conclusion}

A possible mechanism of the rightward inflation phenomena of whistler traces displayed on the sonagram is studied in this paper and the experiments using natural and artificial whistlers described above may be summarized as follows. 
(1) In the quick fading of a whistler wave, the inflation of the width of a trace on the sonagram to the right side could be produced by the resonance echo in the high-Q bandpass filter mounted on a spectrograph of heterodyne type.

(2) The rightward inflation phenomena of semi-circular shape is caused when the amplitude ratio of two or more waves that make fading with each other is less than 0.5 and the delay time may be around $2 \mathrm{~ms}$.

(3) The rightward inflation of the trace becomes clearer as the fading frequency becomes higher.

(4) As the amplitude of two whistler waves becomes closer to each other, the trace shows the structure like a train of patches or a banded type.

(5) The width of the measured whistler trace is wider than the artificial one on the same sonagraph. This difference might be caused by the perturbation of the propagation constant in a whistler duct in the magnetosphere.

Acknowledgments. Whistler data analyzed in this paper were obtained with the Stanford University ELF/VLF Radiometer which was installed at the Earth Observatory, Kochi University. The authors thank Dr. A. C. Fraser-Smith and Prof. R. A. Helliwell for their cooperation in this project. This research is supported by the Grant-in-Aid for Special Project Research, The Ministry of Education, Science and Culture. Thanks are also due to Hitachi Communication Systems, Incorporated for their support in part.

\section{References}

Ohta, K., M. Hayakawa, and H. Eguchi, Ground-based observations of banded whistlers at low latitudes, Res. Lett. Atmos. Electr., 8, 127-136, 1988. (in Japanese)

Ohta, K., M. Hayakawa, and H. Eguchi, On the observation and interpretation of patched structures in the spectra of low latitude whistlers, Trans. IEICE Japan, J72-B- II, 351-358, 1989. (in Japanese)

Paymar, E. M., Banded whistlers observed on OGO-4, Tech. Rep., Stanford University, 3439-1, 1972.

Shaw, R. R. and D. A. Gurnett, Whistlers with harmonic bands caused by multiple stroke lightning, J. Geophys. Res., 76, 1851-1854, 1971.

Yano, S., T. Ogawa, and H. Hagino, Fading phenomena of whistler waves, Res. Lett. Atmos. Electr., 9, 97-107, 1989.

(Received May 28, 1991;

Revised July 3, 1991;

Accepted July 8, 1991.) 